\title{
Effect of Blood's Velocity on Blood Resistivity
}

\author{
Tuqiang Xie, Swee Chuan Tjin, Member, IEEE, Qingping Yang, Member, IEEE, and Seng Lee Ng
}

\begin{abstract}
Blood resistivity is an important quantity whose value influences the results of various methods used in the study of heart and circulation. In this paper, the relationship between blood resistivity and velocity of blood flow was evaluated and analyzed based upon a probe using six-ring electrodes and a circulatory model. The experimental results indicated that the change in blood resistivity was only $\pm 1.1 \%$ when the velocity of blood flow changed from 2.83 to $40 \mathrm{~cm} / \mathrm{s}$ and it rose to $23 \%$ when the velocity was lower than $2.83 \mathrm{~cm} / \mathrm{s}$.
\end{abstract}

Index Terms-Blood flow, blood vessels, electrodes, resistivity measurement, velocity.

\section{INTRODUCTION}

W HEN impedance method is applied to determine the cross-sectional area of a blood vessel, an accurate value of the patient's blood resistivity is required. In fact, the velocity of blood flow in human body varies from patient to patient and even for the same patient. It is very important to understand how the velocity of blood flow influences the blood resistivity and consequently the measurement accuracy of cross-sectional area, so that a compensation scheme may be implemented.

Blood is a suspension of erythrocytes (red cells) of a low electric conductivity in plasma which has a high electrical conductivity. At low frequencies the blood resistivity is a function of the resistivities of erythrocytes and plasma, and of the relative red-cell volume.

Mammalian red cells, human erythrocytes in particular, exhibit a simple architecture. They have no nuclei, and their composition of minerals, proteins, and lipids is relatively well known [1]. They contain about $34 \%$ hemoglobin by weight. Under normal physiological conditions they have the shape of an axisymmetric biconcave disk-shaped body, with an outside diameter of about $8 \mu \mathrm{m}$ and a thickness of $2.5 \mu \mathrm{m}$ at the edges and $1 \mu \mathrm{m}$ in the center [1]. One human red cell in cross section and its approximate dimensions are shown in Fig. 1 [1]. The biconcave shape and the surface area-volume relation allow the membrane to deform without stretching while maintaining a constant surface area.

Because of their biconcave shape and the shape and orientation of the erythrocytes, blood is electrically anisotropic. It is isotropic only when the erythrocytes are homogeneously distributed and in random orientation. In nonhomogeneous

Manuscript received May 18, 1998; revised November 9, 1998. This work was supported by the Ministry of Education, Singapore.

T. Xie and Q. Yang are with the Department of Manufacturing and Engineering Systems, Brunel University, Uxbridge, Middlesex, UB8 3PH, U.K.

S. C. Tjin and S. L. Ng are with the School of Electrical and Electronic Engineering, Nanyang Technological University, Singapore 639798.

Publisher Item Identifier S 0018-9456(98)09776-9.

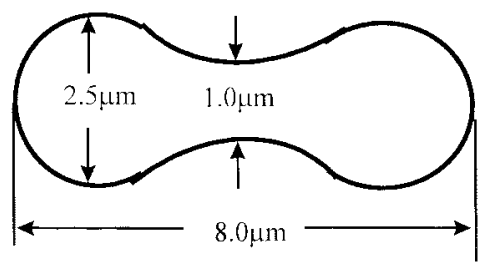

Fig. 1. The red cell in cross section and its nominal dimension.

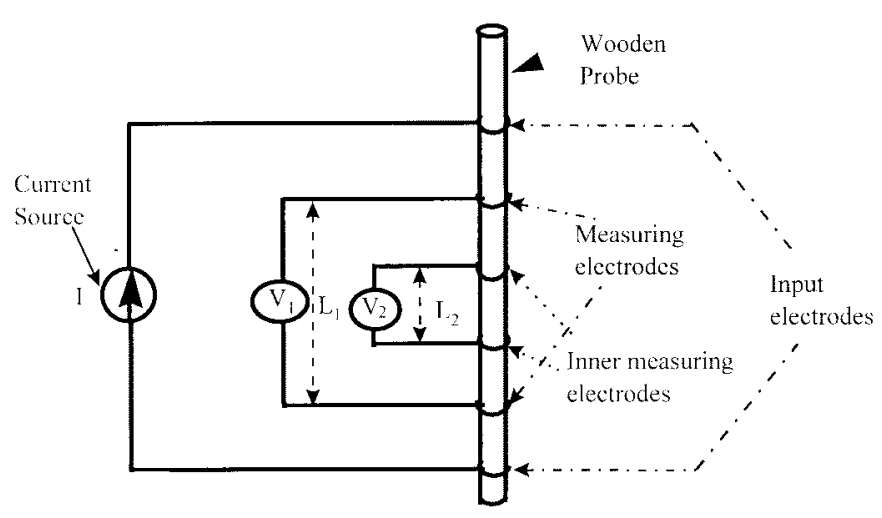

Fig. 2. The six-ring electrodes probe.

one-dimensional situations, it is possible to define an effective resistivity [2]. Such a situation exists in a circular tube with axisymmetric flow and a uniform electric field in the longitudinal direction.

On the other hand, various mechanisms have been proposed to account for changes in the electrical resistivity of blood with flow rate. The changes have been attributed to disaggregation of erythrocytes [3], to electrical "conversion currents" arising from the electrical charge on the erythrocyte surface, to axial accumulation of erythrocytes [3], to reduction-oxidation effects at electrode interfaces, to electrokinetic potentials between the measuring electrodes, and to expansion and contraction of an ionic atmosphere' surrounding the erythrocyte [3].

The maximum velocity of blood flow in the artery is normally about $27-28 \mathrm{~cm} / \mathrm{s}$, but for patients the blood velocity may be out of this range [4]. In order to evaluate and analyze the changes in blood resistivity in relation to blood velocity, a probe with six-ring electrodes (shown in Fig. 2) was employed. The probe was placed within a tube where the blood is flowing at various controlled speeds.

\section{Materials AND MethodS}

Shown in Fig. 3 is a circulatory model, which consists of a heart lung machine, a speedometer, a plastic tube, and a glass column. The heart-lung machine was used to pump the blood 


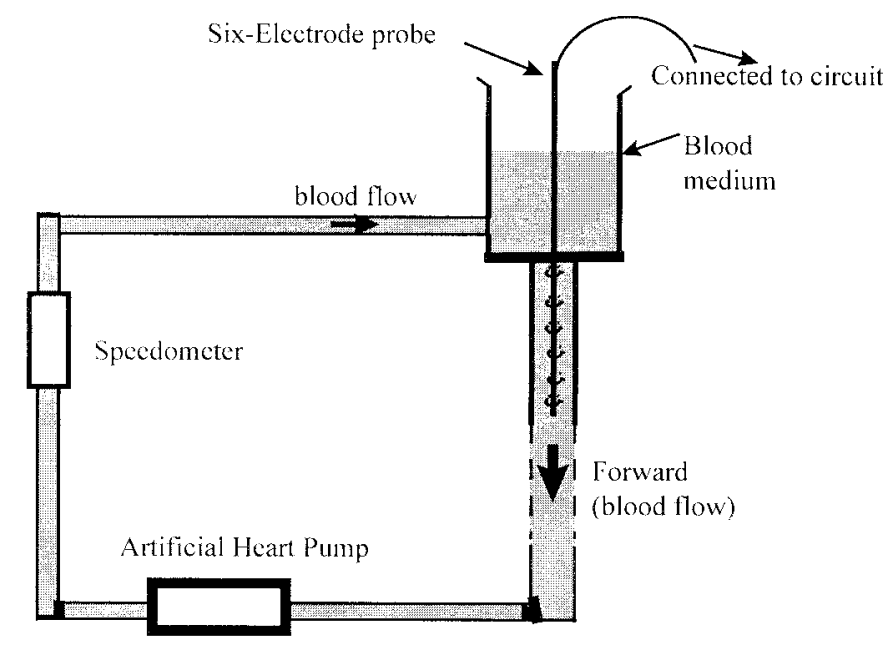

Fig. 3. Setup for measuring the resistivity of flowing blood.

through the tubes without creating excessive turbulence in the flow. A speedometer with error of $1.2 \%$ was included in the system to measure blood flow rate. The velocity of blood flow was calculated based upon the volume flow and tube diameter $(15 \mathrm{~mm})$. The probe with six-ring electrodes was inserted into a tube in the setup. A sinusoidal current of a frequency $3 \mathrm{kHz}$ and a peak amplitude of $17.8 \mu \mathrm{A}$ was applied to the input electrodes of the probe. Two potential differences between the outer and inner two measuring electrodes were amplified, filtered, and measured. These two potential differences and their difference are proportional to the resistivity since the distance between the electrodes, driving current, and the crosssectional area all were fixed. The relationship amongst them can expressed as follows:

$$
\left(V_{1}-V_{2}\right)=\rho \cdot \frac{\left(L_{1}-L_{2}\right) \cdot I}{A}
$$

where $V_{1}, V_{2}$ are two potential differences between the outer and inner two measuring electrodes; the blood resistivity $L_{1}$, $L_{2}$ are the distances between outer measuring electrodes and inner electrodes, respectively; $A$ is the cross-sectional area of plastic tube where the probe was located, and $I$ is the current in input electrodes.

Pig's blood was obtained from the slaughterhouse and heparin was added to it before storing in refrigerator at $4{ }^{\circ} \mathrm{C}$. Prior to experiments, the blood was allowed to warm up to the room temperature $\left(24^{\circ} \mathrm{C}\right)$. It was then poured into the circulator as depicted in Fig. 3. The pig's blood sample was circulated for a short period to ensure that the blood was well mixed. The pump was then turned off and the differential potential, which represents the resistivity of pig's blood in stationary state, was recorded. The velocity of the blood flow was then increased gradually up to $84.92 \mathrm{~cm} / \mathrm{s}(9 \mathrm{~L}$ per min) (the normal human blood flow is around $3 \mathrm{~L}$ per min) [4]. Next, the velocity was decreased gradually and the differential voltage recorded. The experiment was then repeated with the fluid flowing in the reverse direction.

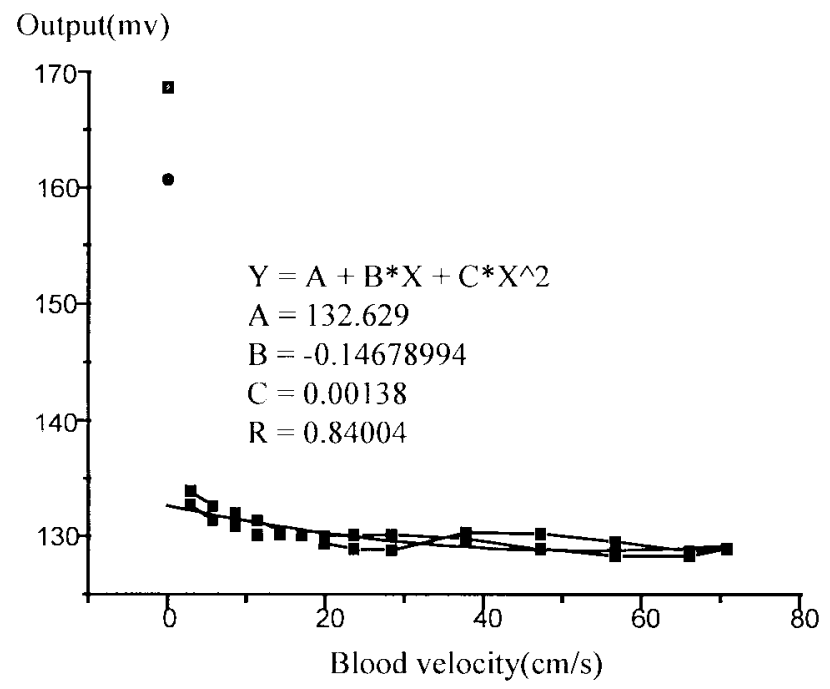

Fig. 4. Voltage change as a function of blood flow velocity. Solution: pig's blood. Direction: forward flow.

\section{RESUlTS AND DisCUSSIONS}

It was pointed out above in Section I that the blood resistivity depends on a number of factors, such as the orientation, disaggregation, and axial accumulation of erythrocytes. Here, only effects of blood flow velocity on measurement of crosssectional area will be discussed due to resistivity change. The results were obtained from the experiments on a large number of samples, and may therefore be considered as typical. When the pig's blood in the circulatory model flows in the forward direction, the potential differences (output) are proportional to the blood resistivity, as shown in Fig. 4. In order to eliminate the interface impedance on the surface of electrodes, the output in the Fig. 4 is the voltage difference between the two pairs of measuring electrodes, i.e., the outer measuring electrodes and the two inner measuring electrodes.

Fig. 4 indicates that when blood changed from the stationary state to the moving state, the output varied significantly. The potential difference declined abruptly from $168.74 \mathrm{mV}$ at stationary to $133.9 \mathrm{mV}$ at $2.83 \mathrm{~cm} / \mathrm{s}$. This is in agreement with the findings of Dellimore and Gosling [4]. In stationary human blood rouleaux formation occurs [4]. The rouleaux break up at high shear stresses. Dellimore and Gosling suggested that rouleaux formation possibly influences the electrical conductivity of blood at velocities below $0.2 \mathrm{~cm} / \mathrm{s}$ (average shear rate $<2.7 \mathrm{~s}^{-1}$ ) [3]. This velocity of rouleaux formation is below our measurements range due to the limitation of the velocity regulation of the heart-lung machine used. This phenomenon can be ruled out, however, as an important cause of blood resistivity changes in the flowing blood. Another main cause of blood resistivity has been contributed to two physical alterations in distribution of the red blood cells, namely the axial accumulation and the orientation of the axis of symmetry of the cells in the lines of flow. Both of these geometrical changes are functions of the blood velocity. It is obvious that greater changes in the blood resistivity occurred in the velocity range between $0 \mathrm{~cm} / \mathrm{s}$ and $17 \mathrm{~cm} / \mathrm{s}$. An increase in blood velocity above $20 \mathrm{~cm} / \mathrm{s}$ did not cause a noticeable change in blood resistivity. This is because there were less geometrical 


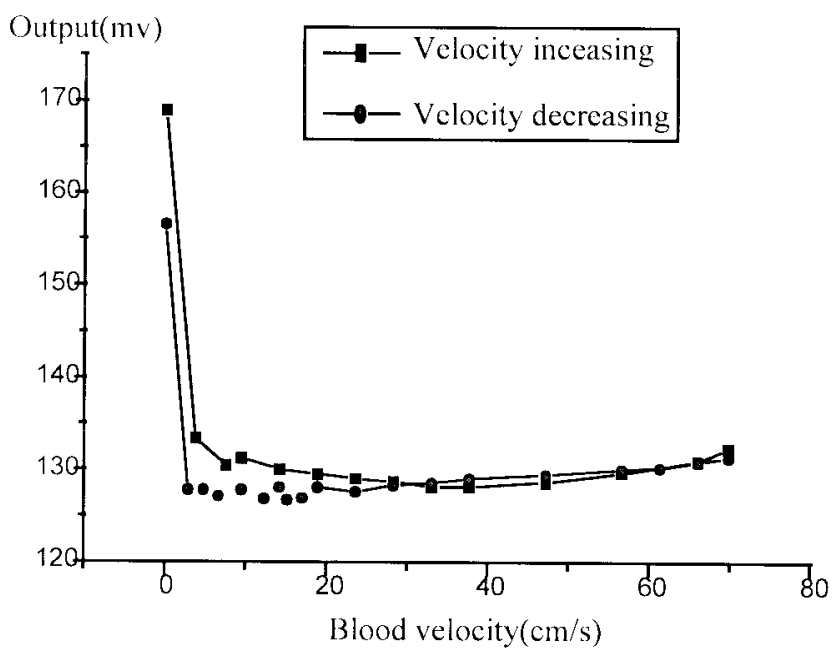

Fig. 5. The voltage change as a function of blood flow velocity. Solution: Pig's blood; Direction: Reverse flow.

changes and hence they contributed less to blood resistivity [5]. On the other hand, as the shear force increased due to the mechanical blood handling in the heart-lung machine used, the red cell deformed from a biconcave shape to prolate spheroid or sphere. With increasing blood velocity, this spheroid shape erythrocytes has less influence on the blood resistivity due to the symmetry of its shape [6].

From the experimental results, the relationship between the output $\left(V_{1}-V_{2}\right)$ and the velocity of blood flow $(v)$, except at the stationary point, may be approximated by a second-order polynomial

$$
\begin{aligned}
\left(V_{1}-V_{2}\right) & =132.629-0.147 \cdot v+0.001382 v^{2} \\
(n & =27, r=0.84004)
\end{aligned}
$$

From (1), the Ohm's law as applied to the six-ring electrodes probe, can also be expressed by

$$
\rho=\frac{A}{I \cdot\left(L_{1}-L_{2}\right)}\left(V_{1}-V_{2}\right)
$$

where $L_{1}-L_{2}, A$, and $I$ are the constants. Hence, the percentage change of blood resistivity due to the velocity change is approximated by

$$
\frac{\Delta \rho}{\rho}=\frac{-0.147 \Delta v+0.00138\left(2 v_{0} \cdot \Delta v+\Delta v^{2}\right)}{132.629-0.147 v_{0}+0.00138 v_{0}^{2}} .
$$

Since the velocity change covered the range of physiological interest, from 2.83 to $40 \mathrm{~cm} / \mathrm{s}, v_{0}$ in (4) was assigned the average velocity of $20 \mathrm{~cm} / \mathrm{s}$ in the range. The relative change of blood resistivity was $\pm 1.1 \%$, even though the velocity of blood flow changes from 2.83 to $40 \mathrm{~cm} / \mathrm{s}$. In the range from 0 to $2.83 \mathrm{~cm} / \mathrm{s}$, however, the relative change of blood resistivity rose to $23.02 \%$ with respect to the velocity change.

The direction of blood flow was changed in the experiment to investigate the effects of flow direction on the measurement results, which are shown in Fig. 5.

The effects of acceleration and deceleration on blood resistivity appeared to be significant in Figs. 4 and 5. This is because the resistance values of suspensions within the flow channel in three mutually orthogonal directions during acceleration are generally more than those during deceleration at the same velocity [3]. In addition, the output voltage of stationary state was higher than that when the blood velocity decelerated from the motion to the stationary state. The observation showed that this difference decreased and disappeared with the time after the velocity of blood was adjusted to zero. This is probably due to the fact that the distribution of the blood and orientation of cells in the tube gradually recovered some time after the moving blood had come to rest.

When the direction of blood flow changed from the forward to reverse, the relationship between the output voltage and the velocity changes little. Although the difference of output voltages during acceleration and deceleration in Fig. 5 are greater than those in Fig. 4, this is insignificant compared with measurement errors.

\section{CONCLUSIONS}

The experimental results have shown that the blood resistivity decreases with the increasing velocity of blood flow. It was observed that the largest change of the blood resistivity occurred in the flow velocities from 0 and $2.83 \mathrm{~cm} / \mathrm{s}$ and the blood resistivity also changed in the velocity range between 2.83 and $17 \mathrm{~cm} / \mathrm{s}$. No significant change, however, was noticed at velocities above $17 \mathrm{~cm} / \mathrm{s}$.

The relationship between the blood resistivity and blood flow velocity was established by curve fitting the experimental results, as shown in (2) and (4). The analysis has shown that a relative change of $\pm 1.1 \%$ in blood resistivity corresponded to a velocity change of $40 \mathrm{~cm} / \mathrm{s}$. But when blood flow velocity changed from 0 to $2.83 \mathrm{~cm} / \mathrm{s}$, the maximum change of blood resistivity may be $23.02 \%$. As a result of this, a compensation scheme may be necessary for blood velocity less than 2.83 $\mathrm{cm} / \mathrm{s}$.

The results also indicated the significant effects of acceleration and deceleration on blood resistivity, but the forward and reverse flows produce the very similar effects.

\section{ACKNOWLEDGMENT}

The authors would like to thank S. Xiaoming for his assistance. The technicians of Photonocs Lab, School of EEE, Nanyang Technological University, provided much assistance during our study.

\section{REFERENCES}

[1] R. Skalak and S. Chien, Handbook of Bioengineering. New York: McGraw-Hill, 1987, p. 12.3 .

[2] K. R. Visser, "Electric conductivity of stationary and flowing human blood at low frequencies," Med. Biol. Eng. Comput., vol. 30, 1992, pp. 636-640.

[3] J. W. Dellimore and R. G. Gosling, "Change in blood conductivity with flow rate," Med. Biol. Eng., vol. 13, pp. 904-913, 1975.

[4] D. A. McDonald, McDonald's Blood Flow in Arteries, Theoretic, Experimental and Clinical Principles. London, U.K.: Lea \& Febiger, 1990, p. 60 .

[5] F. M. Liebman, J. Pearl, and S. Bagno, "The electrical conductance properties of the blood in motion," Phys. Med. Biol., vol. 7, pp. 177-194, 1962. 
[6] R. H. Edgerton, "Conductivity of sheared suspensions of ellipsoidal particles with application to blood flow," IEEE Trans. Biomed. Eng., vol. BME-21, no. 1, pp. 33-43, 1974.

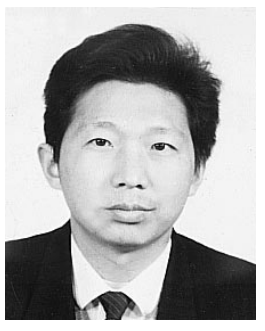

Tuqiang Xie received the B.S. degree in measurement technology and instrumentation in 1982 and the M.S. degree in industrial automation in 1987 from Huazhong University of Science and Technology, China, and the M.Eng. degree from the School of Electrical and Electronic Engineering, Nanyang Technological University, Singapore, in 1997 . He is currently studying and conducting research into metrology and inspection systems for the Ph.D. degree in Department of Manufacturing and Engineering, Brunel University, U.K.

He finished ten projects for industry and published some 20 papers in the field of measurement, instrumentation, automatic control, and image processing.

Swee Chuan Tjin (M'93) received the B.Sc. (Honors Class 1) degree in physics from the University of New England, New South Wales, Australia, in 1987 and the Ph.D. degree from Department of Medicine, University of Tasmania, Tasmania, Australia, in 1992.

$\mathrm{He}$ is currently a Senior Lecturer in the School of Electrical and Electronic Engineering, Nangyang Technological University, Singapore. His research interests are fiber optic sensors, biosensors, and biomedical engineering.

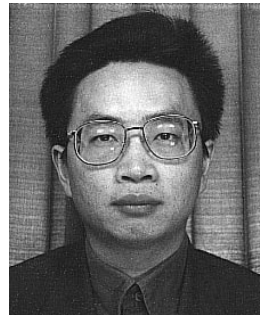

Qingping Yang (M'97) received the Diploma in instrumentation and measurement from Chengdu Aeronautical Polytechnic, Chengdu, China, in 1983. He was awarded a scholarship to study at Brune University, U.K., in 1988 and received the Ph.D. degree in 1992.

He was then an Assistant Engineer, Deputy Head of Department of Measurement and Testing, in the Aircraft Structural Strength Research Institute (Ministry of Aerospace), Shaanxi Province, China His current research interests include dimensional metrology, advanced sensors and transducers, and virtual instrumentation and measurement science. He has published more then 30 papers in these areas.

Seng Lee Ng received the B.Eng. and M.Eng degrees in electrical engineering from Nanyang Technological University (NTU), Singapore, in 1995 and 1998, respectively.

$\mathrm{He}$ is currently a Research Associate in the School of Electrical and Electronic Engineering, NTU. His research interests are photonics devices, fiber optic sensors, and biomedical engineering. 